Published in final edited form as:

Cancer Res. 2013 December 15; 73(24): 7254-7264. doi:10.1158/0008-5472.CAN-13-0750.

\title{
Lenalidomide inhibits lymphangiogenesis in preclinical models of mantle cell lymphoma
}

\author{
Kai Song ${ }^{1}$, Brett H. Herzog ${ }^{1}$, Minjia Sheng ${ }^{1,2}$, Jianxin Fu' ${ }^{1,3,4}$, J. Michael McDaniel ${ }^{1}$, Jia \\ Ruan $^{4,5}$, and Lijun Xia ${ }^{1,3,4,6}$ \\ ${ }^{1}$ Cardiovascular Biology Research Program, Oklahoma Medical Research Foundation, Oklahoma \\ City, Oklahoma \\ ${ }^{2}$ China-Japan Union Hospital of Jilin University, Changchun, China \\ ${ }^{3}$ Key Laboratory of Thrombosis and Hemostasis of Ministry of Health, The First Affiliated Hospital \\ of Soochow University, Suzhou, China \\ ${ }^{4}$ Jiangsu Institute of Hematology, The First Affiliated Hospital of Soochow University, Suzhou, \\ China
}

${ }^{5}$ Center for Lymphoma and Myeloma, Department of Medicine, Weill Cornell Medical College and New York-Presbyterian Hospital, New York City, New York

${ }^{6}$ Department of Biochemistry and Molecular Biology, University of Oklahoma Health Sciences Center, Oklahoma City, Oklahoma

\section{Abstract}

Lymphomas originate in and spread primarily along the lymphatic system. However, whether lymphatic vessels contribute to the growth and spreading of lymphomas is largely unclear. Mantle cell lymphoma (MCL) represents an aggressive non-Hodgkin's lymphoma. We found that MCL exhibited abundant intratumor lymphatic vessels. Our results demonstrated that the immunomodulatory drug lenalidomide potently inhibited the growth and dissemination of MCL in a xenograft MCL mouse model, at least in part, by inhibiting functional tumor lymphangiogenesis. Significant numbers of tumor-associated macrophages expressing vascular endothelial growth factor-C were found in both human MCL and mouse MCL xenograft samples. Lenalidomide treatment resulted in a significant reduction in the number of MCL-associated macrophages. In addition, in vivo depletion of monocytes/macrophages impaired functional tumor lymphangiogenesis and inhibited MCL growth and dissemination. Taken together, our results indicate that tumor lymphangiogenesis contributes to the progression of MCL and that lenalidomide is effective in decreasing MCL growth and metastasis most likely by inhibiting recruitment of MCL-associated macrophages.

Corresponding Authors: Lijun Xia, Cardiovascular Biology Research Program, Oklahoma Medical Research Foundation, 825 N.E. 13th Street, Oklahoma City, OK 73104, USA. Phone: 405-271-7892; Fax: 405-271-3137; lijun-xia@ omrf.org; or Jia Ruan, Center for Lymphoma and Myeloma, Division of Hematology/Oncology, Department of Medicine, Weill Cornell Medical College, 1300 York Avenue, New York, NY 10065, USA, Tel: 646-962-2064, Fax: 646-962-1605, jruan@med.cornell.edu.

K. Song and B. Herzog contributed equally to this work.

Authorship: K.S. designed the study, performed experiments, and drafted the manuscript. B.H. performed experiments and helped draft the manuscript. M.M., M.S., and J.F. helped establish the animal models. J.R. and L.X. designed experiments, supervised the research, and wrote the manuscript.

Conflict of interest: Lenalidomide was provided by Celgene under a material transfer agreement. However, the authors declare no conflict of interest. 


\section{Keywords}

lymphangiogenesis; mantle cell lymphoma; lenalidomide; macrophage; metastasis

\section{Introduction}

Lymphomas are a heterogeneous group of lymphoid malignancies that originate in the lymphatic system (1). However, whether lymphatic vessels contribute to the growth and spreading of lymphomas is largely unclear. Increased expression of the lymphangiogenic factor, vascular endothelial growth factor-C (VEGF-C), and increased lymphatic vessel density have been found in several lymphoma subtypes, including mantle cell lymphoma (MCL), an aggressive form of lymphoma (2). MCL is the most difficult type of lymphoma to treat, which is due in part to its frequent dissemination (3). It is unclear whether and how lymphangiogenesis contributes to the growth and dissemination of MCL.

Recent clinical studies have indicated that the immunomodulatory compound lenalidomide (LEN), either alone or in combination with the CD20 antibody rituximab, has significant effects in non-Hodgkin's lymphoma including MCL (4-7). Multiple anti-tumor mechanisms of action of LEN have been implicated, including immunomodulatory, anti-inflammatory, anti-proliferative, and anti-angiogenic effects (8-13). However, few studies have explored whether LEN affects tumor growth and spreading by inhibiting lymphangiogenesis $(14,15)$.

In our current study, abundant intratumor lymphatic vessels were found in MCL samples from patients and in an experimental mouse MCL xenograft lymphoma model. LEN blocked tumor lymphangiogenesis and inhibited the initiation, progression, and dissemination of MCL in mouse MCL xenografts. We found large numbers of VEGF- $\mathrm{C}^{+}$macrophages in MCL tumor samples from both patients and mice, suggesting that MCL-associated macrophages play an important role in the induction of lymphangiogenesis (16-18). Consistent with this hypothesis, LEN reduced the number of macrophages in MCL. In addition, depletion of monocytes significantly inhibited lymphangiogenesis in MCL tumors and retarded MCL progression and dissemination in the mouse xenograft MCL model. These results indicate that tumor lymphangiogenesis contributes to the progression of MCL and that LEN decreases MCL growth and metastasis most likely by inhibiting recruitment of MCL-associated macrophages.

\section{Materials and Methods}

\section{Cells and drugs}

Mino cells, a well-established cell line derived from MCL patients with verified identity (19), were purchased from ATCC. The cell was tested (by Charles River) free of specific pathogens and maintained in RPMI-1640 (ATCC) supplemented with $20 \%$ heat-inactivated fetal bovine serum and $1 \%$ fungizone, penicillin and streptomycin (Sigma). LEN was provided by Celgene and dissolved in dimethylsulfoxide (DMSO) at a concentration of 250 $\mathrm{mg} / \mathrm{mL}$ as a stock solution. For in vivo injection, LEN was prepared as a $2.5 \mathrm{mg} / \mathrm{mL}$ solution in phosphate-buffered saline (PBS) containing 1\% DMSO (20).

\section{Xenograft mouse models of MCL}

Eight-week-old male immunodeficient NOD.Cg-Prkdc ${ }^{\mathrm{scid}} \mathrm{I} 12 \mathrm{r}_{\mathrm{g}}{ }^{\mathrm{tm} 1 \mathrm{Wj} 1} / \mathrm{SzJ}$ mice (NSG, Jackson Laboratory) were housed in a specific pathogen free mouse facility (21). Mino cells $\left(6 \times 10^{6} / 100 \mu \mathrm{L}\right)$ were injected subcutaneously into both flanks of each NSG mouse near the inguinal lymph node regions. When palpable tumors developed (10 days after inoculation of Mino cells), the mice were randomized into two groups and injected intraperitoneally (i.p.) 
with either LEN (50 mg/kg) or vehicle (1\% DMSO in PBS, sham treated) (10) and were subsequently given a daily i.p. injection of the same amount of LEN or vehicle control for 4 days (short-term treatment) or 21 days (long-term treatment), respectively. Primary tumor volume was measured and calculated as length $\times$ width $^{2} \times 0.5$. At the end of the treatment, mice were sacrificed, and tumors, adjacent lymph nodes, lungs, spleen, liver, and intestine were carefully removed for morphological analysis and immunostaining. All animal experiments were approved by the animal care and use committee of the Oklahoma Medical Research Foundation.

\section{Lymphangiography}

To image the lymph flow from the inguinal to axillary lymph nodes, $20 \mu \mathrm{L}$ Evans blue dye $(0.2 \%$, Sigma) was injected into the hindlimb footpad of each mouse. Forty-five minutes after injection, the mice were sacrificed, and lymphangiography was carried out using a dissection microscope. To determine which lymphatic vessels drained the MCL implanted in the inguinal lymph nodal region, the tumor-bearing mice were anesthetized with isoflurane (E-Z anesthesia system, Euthanex Corporation). A small incision was made in the ventral side of the skin to expose the tumor. FITC-dextran $\left(1.5 \times 10^{6} \mathrm{kDa} \mathrm{MW}\right.$, Sigma $)$ was then slowly injected into the tumor. The incision was closed using sutures. Two hours after injection, a skin flap was made with the tumor near the inguinal lymph nodal areas attached, and draining lymphatic vessels were imaged using an Olympus SZX12 dissection fluorescence microscope.

\section{Immunofluorescent staining}

For immunofluorescent staining of cryosections, tissues were fixed in $4 \%$ paraformaldehyde and embedded in OCT compound (Sakura Finetek). Cryosections ( $20 \mu \mathrm{m}$ in thickness) were blocked with $0.3 \%$ Triton X-100, 3\% donkey serum, 3\% goat serum, and 3\% bovine serum albumin (BSA) in PBS and incubated overnight at $4{ }^{\circ} \mathrm{C}$ with the indicated combinations of the following primary antibodies: Syrian hamster anti-podoplanin (clone 8.1.1), rat antimouse CD31 (clone MEC13.3; BD Biosciences), rat anti-mouse CD105 (clone MJ7/18; eBioscience), rat anti-mouse F4/80 (Life Technologies), biotinylated rat anti-mouse CD11b (clone M1/70; BD Biosciences), biotinylated goat anti-LYVE-1 (clone BAF2125; R\&D Systems), mouse anti-human CD68 (clone KP1; eBioscience), rabbit anti-VEGF-C (Abcam), and rat anti-human CD20 (clone L26; Abcam). After washing in PBS, sections were incubated with the appropriate secondary antibodies conjugated to AlexaFluor (AF)-488, DyLight (DL)-549, or DL-649 (Jackson ImmunoResearch). The sections were mounted with mounting medium with or without 4,6 diamidino-2-phenylindole (DAPI, Invitrogen) and analyzed with confocal scanning microscopy using a DSU spinning-disk head mounted on an Olympus IX81 inverted microscope with a Hamamatsu ORCA-R ${ }^{2}$ camera. Images were analyzed using Slidebook 5.0 (Intelligent Imaging Innovations) and are shown as maximum intensity projections of the $z$-stacks. For quantification of positive staining with the lymphatic marker LYVE-1 and the pan-endothelial marker CD31, a minimum of six randomly chosen fields (10x magnification) of each tumor region (peri, peripheral, and central region) were evaluated (22). For whole-mount staining, the fixed tissues were washed and blocked with $0.3 \%$ Triton X-100, 3\% donkey serum, 3\% goat serum, and 3\% BSA in PBS and immunostained using the following primary antibodies: Syrian hamster anti-mouse podoplanin (clone 8.1.1) and rat anti-CD20 (clone L26; Abcam). Goat $\mathrm{F}\left(\mathrm{ab}^{\prime}\right) 2$ anti-Syrian hamster IgG conjugated to DL-649 or donkey anti-rat IgG conjugated to DL-549 were used as secondary antibodies. 


\section{Histology and immunohistochemical staining}

Paraffin-embedded tissue sections ( $5 \mu \mathrm{m}$ in thickness) were stained with hematoxylin and eosin (H\&E) or were immunohistochemically stained (IHC). IHC was performed as follows: sections were deparaffinized and rehydrated, endogenous peroxidase activity was quenched with $3 \% \mathrm{H}_{2} \mathrm{O}_{2}$ for 10 minutes, antigen retrieval was performed using antigen unmasking solution (Vector Laboratories), and nonspecific binding was blocked with non-specific protein block (Dako) for 1 hour. The slides were incubated with primary antibody against podoplanin (clone D2-40; AngioBio), CD34 (Dako), or VEGFR-3 (Dako) at $4^{\circ} \mathrm{C}$ overnight. After washing, sections were incubated with anti-Syrian hamster secondary antibody conjugated to horseradish peroxidase. Immunoreactivity was visualized using a peroxidasediaminobenzidine kit (Vector Laboratories).

\section{Immunoblotting}

Tumor tissue lysates were used. For immunoblotting, anti-podoplanin (clone 8.1.1), antiProx1 (Abcam), anti-VEGFR-2 (R\&D Systems), anti-VEGFR-3 (R\&D Systems), anti-CCL5 (Cell Signaling), and anti-GAPDH (Cell Signaling) were each used at a dilution of 1:1000. Horseradish peroxidase-conjugated anti-rabbit, anti-Syrian hamster, anti-goat, or antimouse secondary antibodies (Jackson ImmunoResearch) were each used at a 1:5000 dilution. The membranes were developed with SuperSignal West Pico Kit (Thermo Scientific).

\section{Real-time PCR analysis}

Total RNA was extracted from MCL xenografts or cultured Mino cells using RNeasy Plus Mini Kit (Qiagen), according to the manufacturer's instructions. Total RNA was reversely transcribed to cDNA using SuperScriptII Reverse Transcriptase (Life Technologies) with oligo $(\mathrm{dT})_{20}$ primers. Real-time PCR was carried out using $\mathrm{RT}^{2}$ Fast SYBR Green Mastermix (SABiosciences) with specific primer sets $5^{\prime}$ GCTGTCATCCTCATTGCTACTG-3' ${ }^{\prime}$ and $5^{\prime}$-TGGTGTAGAAATACTCCTT GATGTG-3'for human CCL5 (Integrated DNA Technologies). Amplification and detection of mRNA were performed using the CFX96 Real-Time Detection System (Bio-Rad) according to the manufacturer's instructions. To standardize mRNA concentrations, transcript levels of 18S RNA were determined in parallel for each sample, and relative transcript levels were corrected by normalization based on $18 \mathrm{~S}$ transcripts.

\section{Cell migration assay}

Mino cells were cultured in the presence or absence of $10 \mu \mathrm{M}$ LEN for 3 days (23). Same amounts of pre-treated Mino cells $\left(5.4 \times 10^{5} / \mathrm{ml}\right)$ were washed with Hank's balanced salt solution and then cultured with serum-free media for 24 hours. Conditioned media was collected and added to the lower compartment of a transwell ( $5 \mu \mathrm{m}$ pore size, Corning). $10^{5}$ of starved murine macrophages, which were isolated from bone marrow of NSG mice and maintained in RPMI1640 medium containing 20\% FBS and $10 \mathrm{ng} / \mathrm{ml}$ M-CSF (R\&D Systems), were seeded in the upper compartment of a transwell. In some experiments, a neutralizing antibody against CCL5 or isotype control ( $16 \mu \mathrm{g} / \mathrm{ml}$, R\&D Systems) was added to the conditioned media from sham-treated Mino cells. After 4 hours at $37^{\circ} \mathrm{C}$, migrated macrophages were stained and quantified.

\section{Macrophage depletion}

MCL tumor-bearing NSG mice were intravenously administered $100 \mu \mathrm{L}$ plain liposomes (control) or clodronate-encapsulated liposomes (clodroplip, FormuMax), which deplete monocytes/macrophages, every 4 days for 16 days to determine whether macrophages are indispensible for tumor lymphangiogenesis. The efficiency of macrophage depletion was 
measured using flow cytometry of peripheral whole blood that had been stained with antiCD115 (clone AFS98; eBioscience) 5 days after initial administration of liposomes.

\section{Statistical analysis}

Statistical analysis was performed using Student's $t$-test. Differences were considered statistically significant at $P<0.05$.

\section{Results}

\section{MCL tumors exhibit abundant intratumor lymphatic vessels}

To explore the role of lymphatic vessels in MCL development, we characterized the lymphatic vessel density of primary tumors from MCL patient samples and mouse MCL xenografts. Immunostaining for VEGFR-3 and podoplanin, which are lymphatic vessel markers, revealed abundant intratumor lymphatic vessels in human MCL samples (Fig. 1A).

To determine whether intratumor lymphatic vascular density correlates with MCL development, we analyzed different regions (peri-tumor, peripheral and central tumor regions, Fig. 1B) of mouse xenograft MCL tumors using antibodies to LYVE-1, podoplanin, and/or VEGFR-3, three lymphatic vessel markers, and CD31, a pan-endothelial marker. Our results demonstrated that MCL tumors contained increased number of LYVE- $1^{+}$, podoplanin ${ }^{+}$, and CD31 $1^{\text {low }}$ lymphatic vessels in the peri- and peripheral regions of the tumor (Fig. 1C-D and data not shown) in comparison with the lymphatic vascular density in the negative controls (Supplementary Fig. S1A-B), which was the mantle zone of normal nonreactive lymph nodes (24). The lymphatic vessels in the peripheral regions appeared to have open lumens (Fig. 1C; Supplementary Fig. S1C). Most lymphatic vessels were found in the tumor peripheral region (Fig. 1E), as measured by the depth of lymphatic vessel infiltration (25), and the central region rarely had any lymphatic vessels.

\section{LEN inhibits lymphangiogenesis in MCL}

Clinical trials have shown that LEN has significant activities in MCL (6). However, whether LEN inhibits lymphangiogenesis is unclear. Thus, we investigated whether LEN treatment affects MCL tumor lymphangiogenesis in the mouse xenograft MCL model. We observed a dramatic reduction in lymphatic vessel density (Fig. 1C-D) and lymphatic vessel depth of infiltration (Fig. 1C-E) in LEN-treated tumors as compared with sham-treated tumors. To further corroborate the effects of LEN on lymphangiogenesis in MCL, we examined the levels of lymphatic markers in MCL tumors with immunoblotting. Consistent with reduced lymphatic vessel density in MCL tumors after LEN treatment, western blotting detected reduced levels of murine Prox-1, podoplanin, and VEGFR-3, three lymphatic vessels associated proteins, in LEN-treated tumor samples as compared with sham-treated samples (Fig. 1F).

\section{LEN treatment inhibits tumor growth and dissemination in a xenograft mouse model of MCL}

To determine whether LEN affects the progression of MCL in our MCL xenograft model, we examined development of LEN- or sham-treated tumors. Gross morphological analysis revealed that LEN treatment resulted in significant growth retardation of MCL xenografts as compared with the sham-treated group (Fig. 2A-B).

To determine whether LEN affects MCL dissemination, we injected Mino cells into the inguinal lymph nodal region (Fig. 2C) of NSG mice to determine whether the tumor cells spread to axillary lymph nodes, which drain the inguinal region. Thirty-five days after tumor cell injection, gross analysis revealed that axillary lymph nodes were significantly enlarged 
in sham-treated MCL tumor-bearing mice (Fig. 2D). In contrast, few LEN-treated, MCL tumor-bearing mice had enlarged axillary lymph nodes. Since the NSG mice do not have lymphocytes, the enlarged axillary lymph nodes in sham-treated mice were primarily resulted from disseminated MCL cells and infiltrated inflammatory cells as demonstrated by histology and immunofluorescence staining (Fig. 2E, F and Fig. 4B). In contrast, few tumor cells were detected in the axillary lymph nodes of LEN-treated mice. In addition, significantly more lung dissemination was found in sham-treated mice as compared with the LEN-treated group. In the sham-treated group, MCL spreading was occasionally detected in the spleen and rarely detected in other organs, such as the liver or gastrointestinal tract; it was not detected in these organs in LEN-treated mice (Supplementary Fig. S2).

\section{LEN treatment results in non-functional tumor lymphangiogenesis}

To determine the functionality of tumor lymphatic vessels, we first measured the uptake and transport of intra-tumor-injected FITC-dextran by lymphatic vessels. Immunofluorescence staining revealed that FITC-dextran-filled LYVE- $1^{+}$lymphatic vessels were frequently found in the sham-treated tumors. However, most lymphatic vessels did not contain FITCdextran in LEN-treated tumors (Fig. 3A-B). Confocal imaging analyses of MCL samples demonstrated that human $\mathrm{CD} 20^{+}$tumor cells were frequently found inside lymphatic vascular structures in sham-treated but rarely in LEN-treated tumors (Fig. 3C-D). Together, these data support that LEN treatment results in non-functional tumor lymphangiogenesis that likely inhibits tumor cell dissemination through lymphatic vessels.

\section{LEN inhibits MCL spreading through lymphatic vessels}

We observed that MCL inoculated in the inguinal lymph nodal region often disseminated into the axillary lymph nodes (Fig. 2C-F). Lymphangiography performed with Evans blue dye demonstrated a major lymphatic vessel draining the inguinal lymph nodal region into the axillary lymph nodes (Supplementary Fig. S3). To test whether MCL cells spread through this lymphatic route, we injected FITC-dextran into the interstitial space of MCL tumors that were developed after inoculation of Mino cells in the inguinal lymph nodal region and then monitored the transport of the injected FITC-dextran. We found that, in sham-treated mice, FITC-dextran injected into the MCL tumor filled in the draining collecting lymphatic vessels that lead to the axillary lymph nodes; this did not occur in LENtreated mice (Fig. 3E; Supplementary Fig. S4A). In addition, immunostaining revealed that $\mathrm{CD} 20^{+}$tumor cells were within or associated with LYVE- $1^{+}$tumor peripheral lymphatic vessels (Fig. 3F) and in the lymphatic sinuses of axillary lymph nodes (Supplementary Fig. S4B) of sham-treated mice but were rarely observed in these locations in LEN-treated, MCL tumor-bearing mice (Fig. 3F). Functional lymphangiography demonstrated an equivalent rate of transportation of the Evans blue dye through the collecting lymphatic vessels in LEN-treated mice non-tumor bearing mice, compared with sham-treated group (Supplementary Fig. S3). And no substantial change was observed in lymphatic vessel density in the intestine, lung and skin of mice treated with LEN relative to sham-treated mice (Supplementary Fig. S2). These results suggest that LEN treatment impairs functional tumor lymphangiogenesis but not the function of pre-existing host lymphatic vessels. Collectively, these results support that tumor lymphatic vessels serve as an important route for MCL tumor cell spreading and that LEN inhibits this process.

\section{LEN inhibits tumor lymphangiogenesis by decreasing the number of tumor-associated VEGF-C $\mathrm{C}^{+}$macrophages}

To investigate the mechanisms by which LEN affects tumor lymphangiogenesis, we first examined whether LEN affects the survival, proliferation, and motility of lymphatic endothelial cells, which are essential for lymphangiogenesis. However, despite its multiple anti-tumor activities, LEN did not affect these cellular activities of murine lymphatic 
endothelial cells in vitro (Supplementary Fig. S5). Further analyses indicate that LEN did not significantly alter the expression of genes related to lymphangiogenesis and immune cell trafficking, such as VEGFR1, VEGFR2, VEGFR3, E-selectin, P-selectin, PSGL-1, PIGF-2, and VE-cadherin $(15,26,27)$, in lymphatic endothelial cells (Supplementary Fig. S6A). These results support that directly targeting lymphatic endothelial cells is not a mechanism by which LEN inhibits MCL lymphangiogenesis.

Lymphangiogenic factor VEGF-C derived from tumor-associated macrophage and/or cancer cells are essential for tumor lymphangiogenesis $(28,29)$. We found that VEGF-C was expressed in macrophages but not in tumor cells in human MCL samples $(28.6 \% \pm 2.6 \%$ of macrophages, Fig. 4A), suggesting that macrophages may be critical for lymphangiogenesis in MCL. Consistent with this finding, mouse MCL xenografts also contained large numbers of VEGF-C ${ }^{+}$cells that co-localized with the macrophage markers F4/80 and CD11b (Fig. 4B). In addition, we found that cultured Mino cells did not express VEGF-C under either normal culture condition or after $\mathrm{CoCl}_{2}$ treatment, which induces hypoxic condition (Supplementary Fig. S7A). This suggests that tumor-associated macrophages, but not tumor cells, are the major source of VEGF-C. LEN treatment resulted in reduced expression of VEGF-C mRNA in the MCL xenografts (Supplementary Fig. S7A). Immunofluorescence staining revealed that large numbers of $\mathrm{F} 4 / 80^{+} \mathrm{CD} 11 \mathrm{~b}^{+} \mathrm{VEGF}-\mathrm{C}^{+}$macrophages were detected in the peripheral regions of sham-treated but not LEN-treated tumors (Fig. 4B-C; Supplementary Fig. S7B-C). These results support that LEN attenuates VEGF-C expression by decreasing tumor-associated macrophages.

\section{LEN inhibits the recruitment of tumor-associated macrophages}

Infiltrated monocytes/macrophages play important roles in the promotion of tumor angiogenesis/lymphangiogenesis $(27,30)$. We then asked how LEN inhibits tumorinfiltrating macrophages. Initial semi-quantitative RT-PCR analysis demonstrated that LEN did not significantly alter the expression of genes related to the recruitment of monocytes/ macrophages in macrophages (Supplementary Fig. S6B). Further analyses of sham- or LENtreated primary bone marrow-derived macrophages from NSG mice indicated that LEN did not directly affect the proliferation, survival, and migration of macrophages either (Supplementary Fig. S8).

Tumor cells-derived factors such as chemokines, cytokines, and cytokine-like growth factors are essential for the recruitment of monocytes/macrophages (30). To determine whether LEN inhibits the potency of MCL tumor cells to attract macrophages, we performed a focused gene profiling analysis of sham- or LEN-treated MCL xenograft samples using a human SABiosciences $\mathrm{RT}^{2}$ Profiler ${ }^{\mathrm{TM}}$ Array, which contains 84 key genes central to recruitment and/or activation of immune cells (31-33). This analysis revealed that chemokine CCL5 was ranked on top of genes that were significantly downregulated in LENtreated samples relative to sham-treated (Fig. 4D; Supplementary Fig. S6C). We chose to focus on CCL5 for in depth analysis since it is a key regulator for monocyte/macrophage recruitment $(34,35)$. Quantification of CCL5 by real-time PCR and immunoblotting validated the gene array result (Fig. 4D-E). Consistent with this, conditioned media collected from Mino cell cultured in the presence of LEN significantly decreased the macrophage chemotaxis in a transwell cell migration assay compared to media from sham-treated cells (Fig. 4F). Importantly, macrophage migration was significantly reduced when exposed to Mino cell conditioned media containing a CCL5 neutralizing antibody but not control $\operatorname{IgG}$ (Fig. 4G) (36). These data indicate that LEN inhibits tumor lymphangiogenesis by reducing MCL cells from producing factors such as CCL5 that are important for attracting macrophages. 


\section{Clodronate liposome-mediated macrophage depletion inhibits tumor lymphangiogenesis and retards MCL growth and spreading}

If LEN decreases MCL lymphangiogenesis by inhibiting MCL recruitment of macrophages, depleting macrophages would result in a similar anti-MCL effect. To test this, we depleted monocyte/macrophages in MCL tumor-bearing mice with clodronate encapsulated in liposomes (clodrolip) (37). Flow cytometric analysis of CD115, a monocyte/macrophage marker on peripheral blood cells, demonstrated that clodrolip treatment resulted in an $86.5 \%$ reduction in $\mathrm{CD}_{115^{+}}$cells (Fig. 5A). Consistent with this result, F4/80 staining in clodroliptreated tumors demonstrated a significant reduction in the number of tumor-associated macrophages, which was accompanied by reduced tumor growth (Fig. 5B-C; Supplementary Fig. S9). A significant impairment in the function and the number of intratumor lymphatic vessels after clodrolip treatment was observed as compared with the control group (Fig. 5DF). Moreover, clodrolip-treated mice exhibited reduced spreading of MCL xenografts into the axillary lymph nodes and lungs as compared with the control liposome treatment (Fig. 5G, H and data not shown). Together, these results indicate that clodrolip-mediated depletion of tumor-associated macrophages significantly blocks functional tumor lymphangiogenesis and subsequently impairs the growth and dissemination of MCL.

\section{Discussion}

Lymphangiogenesis during tumor growth and metastasis has been an emerging focus of vascular biology in recent years (27). However, whether and how lymphatic vessels contribute to dissemination of lymphoma remains unclear. Our study provides novel and definitive evidence to support that 1) mantle cell lymphomas in preclinical models have heightened lymphangiogenesis compared to the mantle zone of normal lymph nodes; 2) LEN attenuates lymphatic spreading by reducing functional lymphangiogenesis; and 3) the anti-lymphangiogenic mechanisms of LEN are, at least partly, mediated by reducing the number of tumor-infiltrating macrophages and their production of VEGF-C. To our knowledge, this is the first report on the novel therapeutic anti-lymphangiogenic mechanism of LEN in lymphoma and highlights the potential pathogenic role of lymphangiogenesis in lymphoma progression and dissemination.

Anti-lymphangiogenic agents may have therapeutic potential in a variety of malignancy types. Currently, anti-lymphatic strategies target primarily the signaling induced by VEGF$\mathrm{C}$ and its receptor, VEGFR-3. A VEGFR-3-targeting monoclonal antibody, IMC-3C5, has recently entered phase I clinical trials for patients with advanced solid tumors that are refractory to standard therapy or for which no standard therapy is available (15). Blocking antibodies to neuropilin-2, a co-receptor for VEGF-C, have also shown efficacy in reducing lymphangiogenesis and lung metastasis in animal models $(38,39)$. LEN (Revlimid $\left.{ }^{\mathrm{TM}}\right)$, a derivative of thalidomide, was initially introduced as a treatment for multiple myeloma. Either alone or in combination with other drugs such as rituximab, dexamethasone, and bortezomib, LEN has proven effective in the treatment of MCL in clinical trials $(3,40,41)$. Therefore, it has recently been approved by the FDA for the treatment of patients with relapsed or refractory MCL despite the fact that it may potentially induce secondary cancers such as Hodgkin's lymphoma $(42,43)$. These anti-tumor effects of LEN have been largely attributed to its cytotoxicity directly to MCL cell growth $(10,13)$. Our study provides an additional mechanism of actions for LEN. We show novel evidence supporting that LEN attenuates tumor growth and lymphatic metastasis by reducing functional tumor lymphangiogenesis without affecting pre-existing host lymphatic vasculature. LEN inhibits lymphangiogenesis at the early stage of MCL development (Supplementary Fig. S10), supporting that LEN-mediated reduction of lymphangiogenesis results in suppression of MCL growth and metastasis. In addition to potent inhibition of lymphangiogenesis, LEN treatment causes non-functional angiogenesis in our MCL mouse model (Supplementary 
Fig. S11), which is consistent with the data from a recent phase II clinical study (44).

Therefore, the anti-lymphangiogenesis and anti-angiogenesis effects of LEN may synergistically contribute to its inhibition of MCL growth and spreading.

Current knowledge regarding whether and how lymphatic vessels contribute to metastasis of MCL is limited. The presence and significance of intratumoral lymphatic vessels has been a controversial subject $(14,22,45,46)$. In this study, we identified intratumor lymphatic vessels in peripheral regions of samples from both patients and mice with MCL. These vessels appeared to have open lumens and contain tumor cells. The intratumor lymphatic vessels in the mouse MCL xenografts were functional as shown with FITC-dextran lymphangiography. In addition, we observed MCL tumor cells in the lymphatic vessels that surround the primary tumor and in the axillary lymph nodes of sham-treated mice. However, tumor cells were not detected in these vessels in LEN-treated mice. These results suggest that increased intratumor functional lymphangiogenesis contributes to tumor cell spreading along draining lymphatic vessels to the adjacent lymph nodes and that blocking this process contributes to the mechanisms of the anti-MCL action of LEN.

The tumor microenvironment influences neoplastic progression and growth (32). Non-tumor stromal and infiltrating immune cells, including lymphoma-associated macrophages, have been recognized as important adverse prognostic factors $(31,33)$. Consistent with this, we detected high numbers of lymphoma-associated macrophages in MCL. The CD11 $\mathrm{b}^{+}$ macrophage subset is known to produce large amounts of VEGF-C, which is important for lymphangiogenesis (17). Importantly, LEN treatment reduced the recruitment of tumorinfiltrating macrophages primarily at the tumor peripheral region where tumor lymphangiogenesis is active. Depletion of macrophages reduced tumor growth and metastasis. These results reveal a novel mechanism of action for LEN in MCL treatment and support the importance of the tumor microenvironment in MCL progression.

\section{Supplementary Material}

Refer to Web version on PubMed Central for supplementary material.

\section{Acknowledgments}

We thank Kirk Bergstrom, Reema Biswas, Yanfang Pan, Bojing Shao, Ying Wang, Nan Zhang, and Lili Yu for technical support. Tissue processing was performed in the imaging core facility of the Oklahoma Medical Research Foundation.

Grant Support: This work was supported by grants from the NIH (GM103441 and HL085607), Chinese National Natural Science Foundation (30928010) (to L. Xia); ASCO Career Development Award and NIH grant K08HL091517 (to J. Ruan); Jiangsu Provincial Special Program of Medical Science (BL2012005), Jiangsu Province's Key Medical Center (ZX201102) (to L. Xia, J. Fu, and J. Ruan).

\section{References}

1. Morton LM, Wang SS, Devesa SS, Hartge P, Weisenburger DD, Linet MS. Lymphoma incidence patterns by WHO subtype in the United States, 1992-2001. Blood. 2006; 107:265-76. [PubMed: 16150940]

2. Kadowaki I, Ichinohasama R, Harigae H, Ishizawa K, Okitsu Y, Kameoka J, et al. Accelerated lymphangiogenesis in malignant lymphoma: possible role of VEGF-A and VEGF-C. Br J Haematol. 2005; 130:869-77. [PubMed: 16156857]

3. Jares P, Colomer D, Campo E. Genetic and molecular pathogenesis of mantle cell lymphoma: perspectives for new targeted therapeutics. Nat Rev Cancer. 2007; 7:750-62. [PubMed: 17891190] 
4. Habermann TM, Lossos IS, Justice G, Vose JM, Wiernik PH, McBride K, et al. Lenalidomide oral monotherapy produces a high response rate in patients with relapsed or refractory mantle cell lymphoma. Br J Haematol. 2009; 145:344-9. [PubMed: 19245430]

5. Wang M, Fayad L, Wagner-Bartak N, Zhang L, Hagemeister F, Neelapu SS, et al. Lenalidomide in combination with rituximab for patients with relapsed or refractory mantle-cell lymphoma: a phase 1/2 clinical trial. Lancet Oncol. 2012; 13:716-23. [PubMed: 22677155]

6. Wiernik PH, Lossos IS, Tuscano JM, Justice G, Vose JM, Cole CE, et al. Lenalidomide monotherapy in relapsed or refractory aggressive non-Hodgkin's lymphoma. J Clin Oncol. 2008; 26:4952-7. [PubMed: 18606983]

7. Witzig TE, Wiernik PH, Moore T, Reeder C, Cole C, Justice G, et al. Lenalidomide oral monotherapy produces durable responses in relapsed or refractory indolent non-Hodgkin's Lymphoma. J Clin Oncol. 2009; 27:5404-9. [PubMed: 19805688]

8. Corral LG, Haslett PA, Muller GW, Chen R, Wong LM, Ocampo CJ, et al. Differential cytokine modulation and $\mathrm{T}$ cell activation by two distinct classes of thalidomide analogues that are potent inhibitors of TNF-alpha. J Immunol. 1999; 163:380-6. [PubMed: 10384139]

9. LeBlanc R, Hideshima T, Catley LP, Shringarpure R, Burger R, Mitsiades N, et al. Immunomodulatory drug costimulates T cells via the B7-CD28 pathway. Blood. 2004; 103:178790. [PubMed: 14512311]

10. Qian Z, Zhang L, Cai Z, Sun L, Wang H, Yi Q, et al. Lenalidomide synergizes with dexamethasone to induce growth arrest and apoptosis of mantle cell lymphoma cells in vitro and in vivo. Leuk Res. 2011; 35:380-6. [PubMed: 21047686]

11. Reddy N, Hernandez-Ilizaliturri FJ, Deeb G, Roth M, Vaughn M, Knight J, et al. Immunomodulatory drugs stimulate natural killer-cell function, alter cytokine production by dendritic cells, and inhibit angiogenesis enhancing the anti-tumour activity of rituximab in vivo. Br J Haematol. 2008; 140:36-45. [PubMed: 17995965]

12. Wu L, Adams M, Carter T, Chen R, Muller G, Stirling D, et al. lenalidomide enhances natural killer cell and monocyte-mediated antibody-dependent cellular cytotoxicity of rituximab-treated CD20+ tumor cells. Clin Cancer Res. 2008; 14:4650-7. [PubMed: 18628480]

13. Zhang L, Qian Z, Cai Z, Sun L, Wang H, Bartlett JB, et al. Synergistic antitumor effects of lenalidomide and rituximab on mantle cell lymphoma in vitro and in vivo. Am J Hematol. 2009; 84:553-9. [PubMed: 19565649]

14. Padera TP, Kadambi A, di Tomaso E, Carreira CM, Brown EB, Boucher Y, et al. Lymphatic metastasis in the absence of functional intratumor lymphatics. Science. 2002; 296:1883-6. [PubMed: 11976409]

15. Alitalo K. The lymphatic vasculature in disease. Nat Med. 2011; 17:1371-80. [PubMed: 22064427]

16. Schoppmann SF, Birner P, Stockl J, Kalt R, Ullrich R, Caucig C, et al. Tumor-associated macrophages express lymphatic endothelial growth factors and are related to peritumoral lymphangiogenesis. Am J Pathol. 2002; 161:947-56. [PubMed: 12213723]

17. Kerjaschki D. The crucial role of macrophages in lymphangiogenesis. J Clin Invest. 2005; 115:2316-9. [PubMed: 16138185]

18. Maruyama K, Ii M, Cursiefen C, Jackson DG, Keino H, Tomita M, et al. Inflammation-induced lymphangiogenesis in the cornea arises from CD11b-positive macrophages. J Clin Invest. 2005; 115:2363-72. [PubMed: 16138190]

19. Lai R, McDonnell TJ, O'Connor SL, Medeiros LJ, Oudat R, Keating M, et al. Establishment and characterization of a new mantle cell lymphoma cell line, Mino. Leuk Res. 2002; 26:849-55. [PubMed: 12127561]

20. Pellagatti A, Jadersten M, Forsblom AM, Cattan H, Christensson B, Emanuelsson EK, et al. Lenalidomide inhibits the malignant clone and up-regulates the SPARC gene mapping to the commonly deleted region in 5q- syndrome patients. Proc Natl Acad Sci U S A. 2007; 104:1140611. [PubMed: 17576924]

21. Shultz LD, Lyons BL, Burzenski LM, Gott B, Chen X, Chaleff S, et al. Human lymphoid and myeloid cell development in NOD/LtSz-scid IL2R gamma null mice engrafted with mobilized human hemopoietic stem cells. J Immunol. 2005; 174:6477-89. [PubMed: 15879151] 
22. Alitalo A, Detmar M. Interaction of tumor cells and lymphatic vessels in cancer progression. Oncogene. 2012; 31:4499-508. [PubMed: 22179834]

23. Schulz A, Durr C, Zenz T, Dohner H, Stilgenbauer S, Lichter P, et al. Lenalidomide reduces survival of chronic lymphocytic leukemia cells in primary cocultures by altering the myeloid microenvironment. Blood. 2013; 121:2503-11. [PubMed: 23349394]

24. Barista I, Romaguera JE, Cabanillas F. Mantle-cell lymphoma. Lancet Oncol. 2001; 2:141-8. [PubMed: 11902564]

25. Skobe M, Hawighorst T, Jackson DG, Prevo R, Janes L, Velasco P, et al. Induction of tumor lymphangiogenesis by VEGF-C promotes breast cancer metastasis. Nat Med. 2001; 7:192-8. [PubMed: 11175850]

26. St Hill CA. Interactions between endothelial selectins and cancer cells regulate metastasis. Front Biosci-Landmrk. 2011; 16:3233-51.

27. Tammela T, Alitalo K. Lymphangiogenesis: Molecular mechanisms and future promise. Cell. 2010; 140:460-76. [PubMed: 20178740]

28. Zumsteg A, Baeriswyl V, Imaizumi N, Schwendener R, Ruegg C, Christofori G. Myeloid cells contribute to tumor lymphangiogenesis. PLoS One. 2009; 4:e7067. [PubMed: 19759906]

29. Alitalo K, Tammela T, Petrova TV. Lymphangiogenesis in development and human disease. Nature. 2005; 438:946-53. [PubMed: 16355212]

30. Murdoch C, Muthana M, Coffelt SB, Lewis CE. The role of myeloid cells in the promotion of tumour angiogenesis. Nat Rev Cancer. 2008; 8:618-31. [PubMed: 18633355]

31. Monti S, Savage KJ, Kutok JL, Feuerhake F, Kurtin P, Mihm M, et al. Molecular profiling of diffuse large B-cell lymphoma identifies robust subtypes including one characterized by host inflammatory response. Blood. 2005; 105:1851-61. [PubMed: 15550490]

32. Farinha P, Masoudi H, Skinnider BF, Shumansky K, Spinelli JJ, Gill K, et al. Analysis of multiple biomarkers, shows that lymphoma-associated macrophage (LAM) content is an independent predictor of survival in follicular lymphoma (FL). Blood. 2005; 106:2169-74. [PubMed: 15933054]

33. Dave SS, Wright G, Tan B, Rosenwald A, Gascoyne RD, Chan WC, et al. Prediction of survival in follicular lymphoma based on molecular features of tumor-infiltrating immune cells. N Engl J Med. 2004; 351:2159-69. [PubMed: 15548776]

34. Ek S, Bjorck E, Hogerkorp CM, Nordenskjold M, Porwit-MacDonald A, Borrebaeck CA. Mantle cell lymphomas acquire increased expression of CCL4, CCL5 and 4-1BB-L implicated in cell survival. Int J Cancer. 2006; 118:2092-7. [PubMed: 16287062]

35. Mazur G, Jaskula E, Kryczek I, Dlubek D, Butrym A, Wrobel T, et al. Proinflammatory chemokine gene expression influences survival of patients with non-Hodgkin's lymphoma. Folia Histochem Cytobiol. 2011; 49:240-7. [PubMed: 21744323]

36. Eyman D, Damodarasamy M, Plymate SR, Reed MJ. CCL5 secreted by senescent aged fibroblasts induces proliferation of prostate epithelial cells and expression of genes that modulate angiogenesis. J Cell Physiol. 2009; 220:376-81. [PubMed: 19360811]

37. Zeisberger SM, Odermatt B, Marty C, Zehnder-Fjallman AH, Ballmer-Hofer K, Schwendener RA. Clodronate-liposome-mediated depletion of tumour-associated macrophages: a new and highly effective antiangiogenic therapy approach. Br J Cancer. 2006; 95:272-81. [PubMed: 16832418]

38. Xu Y, Yuan L, Mak J, Pardanaud L, Caunt M, Kasman I, et al. Neuropilin-2 mediates VEGF-Cinduced lymphatic sprouting together with VEGFR3. J Cell Biol. 2010; 188:115-30. [PubMed: 20065093]

39. Caunt M, Mak J, Liang WC, Stawicki S, Pan Q, Tong RK, et al. Blocking neuropilin-2 function inhibits tumor cell metastasis. Cancer cell. 2008; 13:331-42. [PubMed: 18394556]

40. Damaj G, Lefrere F, Delarue R, Varet B, Furman R, Hermine O. Thalidomide therapy induces response in relapsed mantle cell lymphoma. Leukemia. 2003; 17:1914-5. [PubMed: 12970798]

41. Kaufmann H, Raderer M, Wohrer S, Puspok A, Bankier A, Zielinski C, et al. Antitumor activity of rituximab plus thalidomide in patients with relapsed/refractory mantle cell lymphoma. Blood. 2004; 104:2269-71. [PubMed: 15166030]

42. Landgren O, Thomas A, Mailankody S. Myeloma and second primary cancers. N Engl J Med. 2011; 365:2241-2. [PubMed: 22150057] 
43. Pan B, Lentzsch S. The application and biology of immunomodulatory drugs (IMiDs) in cancer. Pharmacol Ther. 2012; 136:56-68. [PubMed: 22796518]

44. Zaja F, De Luca S, Vitolo U, Orsucci L, Levis A, Salvi F, et al. Salvage treatment with lenalidomide and dexamethasone in relapsed/refractory mantle cell lymphoma: clinical results and effects on microenvironment and neo-angiogenic biomarkers. Haematologica. 2012; 97:416-22. [PubMed: 22058200]

45. Cassella M, Skobe M. Lymphatic vessel activation in cancer. Annals of the New York Academy of Sciences. 2002; 979:120-30. [PubMed: 12543722]

46. Das S, Skobe M. Lymphatic vessel activation in cancer. Annals of the New York Academy of Sciences. 2008; 1131:235-41. [PubMed: 18519976] 
A
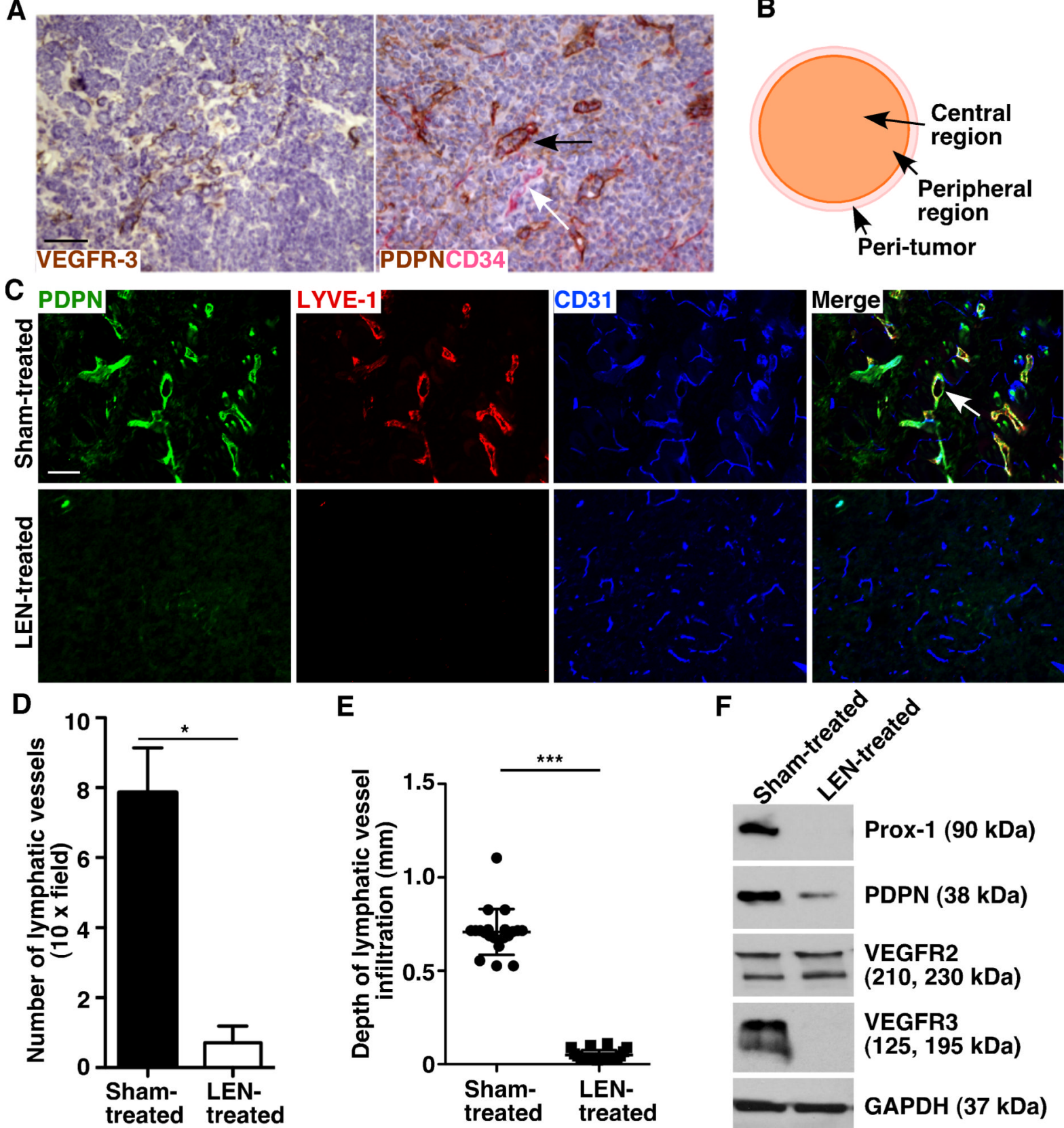

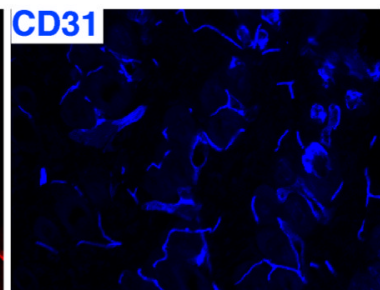

B
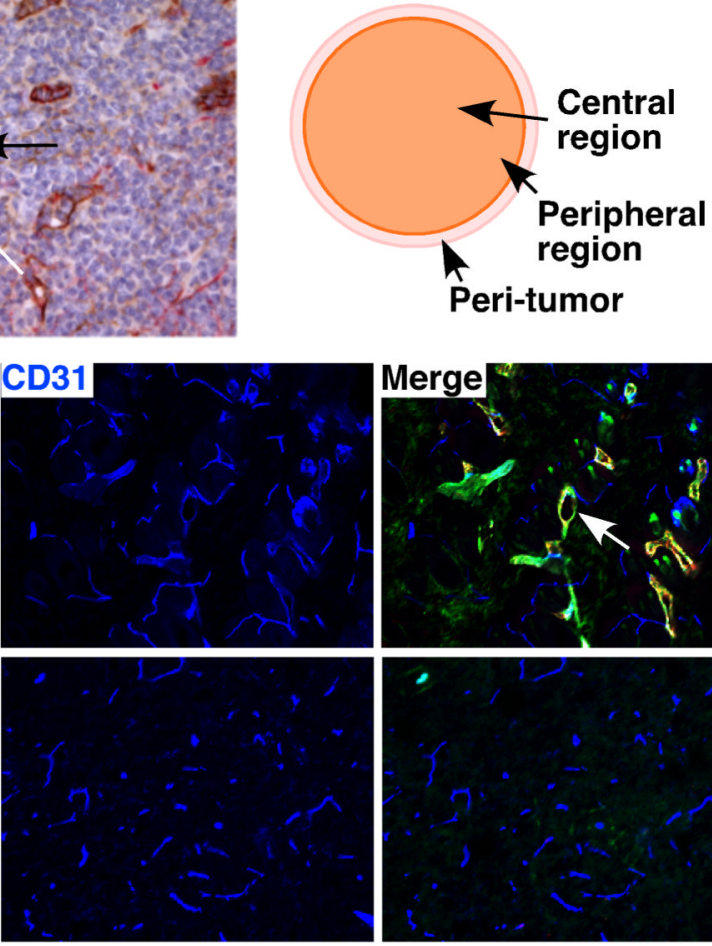

E

$\mathbf{F}$

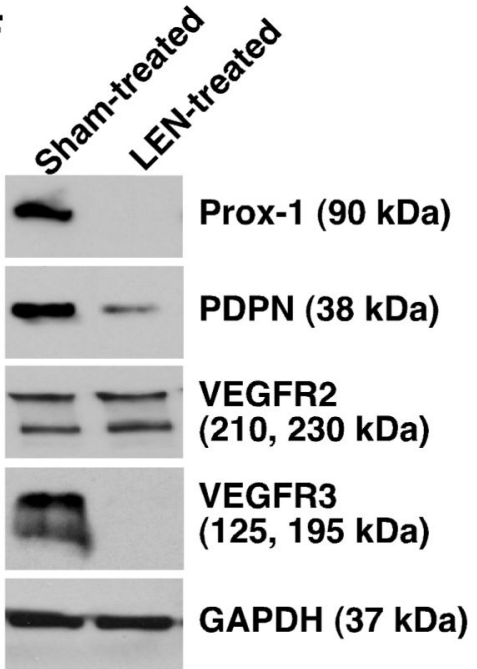

Figure 1.

MCL tumors exhibit abundant intratumor lymphatic vessels, and LEN decreases lymphangiogenesis in MCL. A. Immunohistochemical analysis of two lymphatic markers, VEGFR-3 and podoplanin (PDPN), and a blood vessel marker, CD34, in human MCL patient samples. The black arrow indicates an intratumor lymphatic vessel. The white arrow indicates a blood vessel. B. Schematic diagram showing different tumor regions. C. Immunofluorescence staining for PDPN, LYVE-1, and CD31 in sections of sham- and LENtreated murine MCL tumors. Images were taken from peripheral regions of the tumor. White arrow indicates a LYVE-1 $1^{+}, \mathrm{PDPN}^{+}$, and CD31 ${ }^{\text {low }}$ lymphatic vessel with an open lumen. D. Quantification of LYVE-1 $1^{+}$, CD $31^{+}$lymphatic vessels. Data represent the mean \pm SEM $(n=$ 20). ${ }^{*} P<0.05$. E. Quantification of the depth of infiltrated lymphatic vessels. The depths 
were calculated as the distance from the tumor-host interface to the innermost lymphatic vessels. Data represent the mean $\pm \mathrm{SD}(\mathrm{n}=20) . * * * P<0.001$. F. Immunoblots of Prox-1, PDPN, VEGFR-2, and VEGFR-3 in sham- and LEN-treated mouse MCL tumors. GAPDH is an internal control. Data represent two experiments. Scale bars, $100 \mu \mathrm{m}$. 

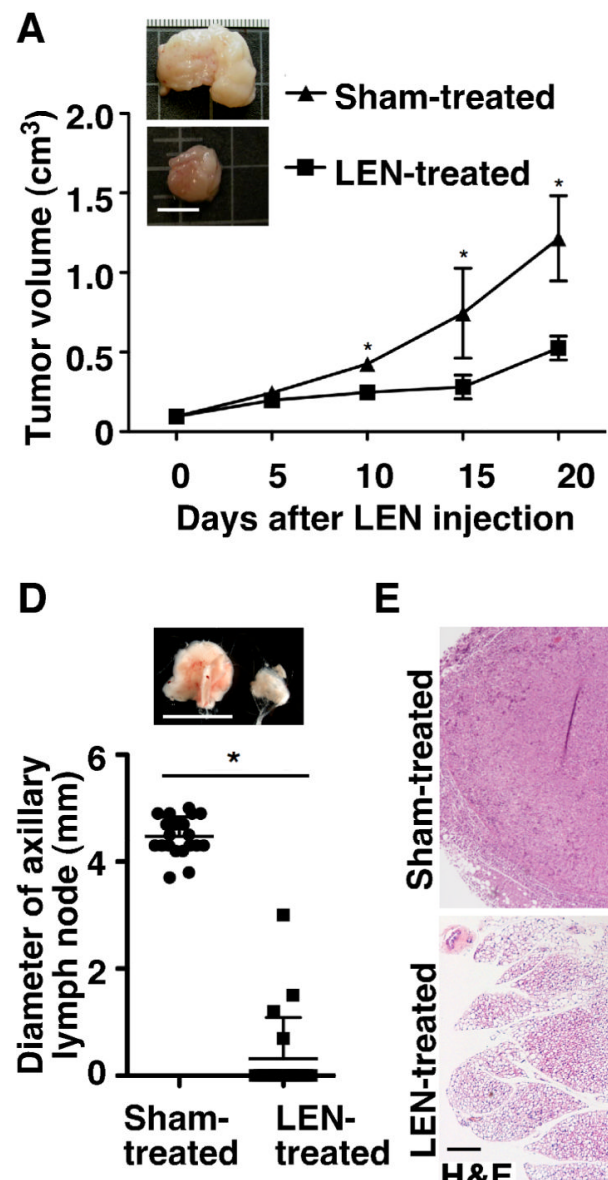

B

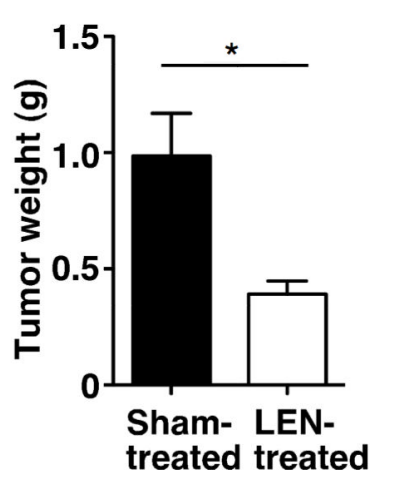

C

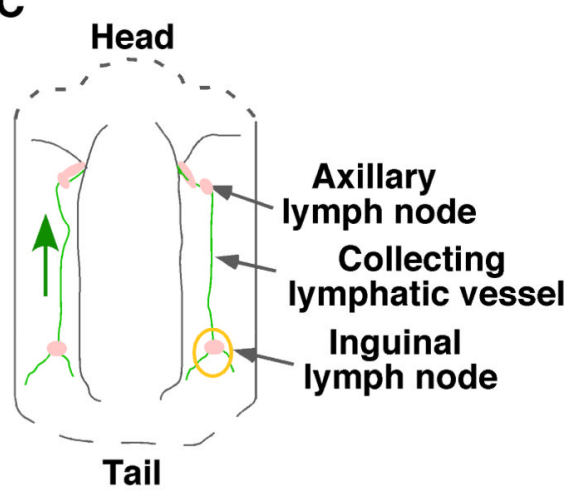

E

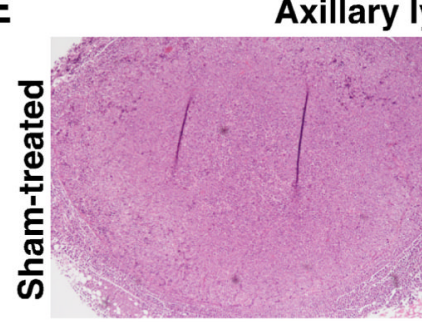

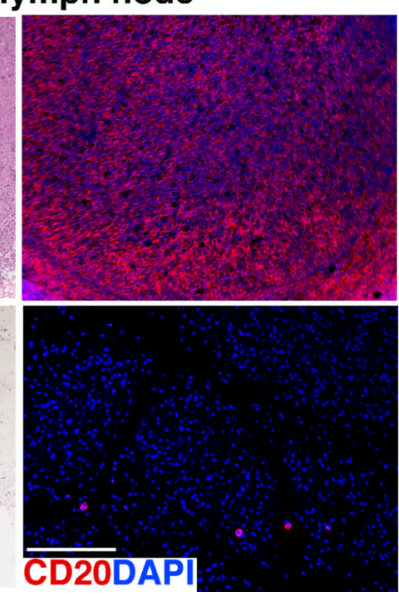

$\mathbf{F}$

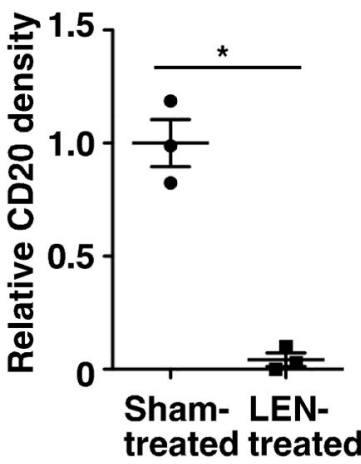

Figure 2.

LEN treatment inhibits MCL growth and dissemination. A. The tumor volume of Mino cellderived MCL mouse xenografts in NSG mice was measured at different times and is presented as the mean \pm SEM $(n=10)$. Scale bar, $10 \mathrm{~mm}$. B. Tumors were weighed at harvest, and data are presented as the mean $\pm \mathrm{SD}(\mathrm{n}=10)$. C. Schematic diagram showing inguinal lymph node, collecting lymphatic vessel, and axillary lymph node in mouse skin flaps. The yellow circle indicates the site of MCL xenograft. Green arrow shows the direction of lymphatic flow. D. Images and diameters of axillary lymph nodes. Data represent the mean $\pm \mathrm{SD}(\mathrm{n}=10)$. Scale bar, $5 \mathrm{~mm}$. E. H\&E and immunostaining of the human B-cell marker, CD20, in sections of axillary lymph nodes from sham- and LENtreated mice. Scale bars, $100 \mu \mathrm{m}$. F. Quantification of disseminated CD20 ${ }^{+}$Mino cells in the axillary lymph nodes using ImageJ. The relative staining density was calculated by comparing CD20 ${ }^{+}$areas per $10 \times$ field of LEN-treated samples with those of sham-treated samples. Data represent three independent experiments (Mean $\pm \mathrm{SEM}, \mathrm{n}=10, * P<0.05$ ). 

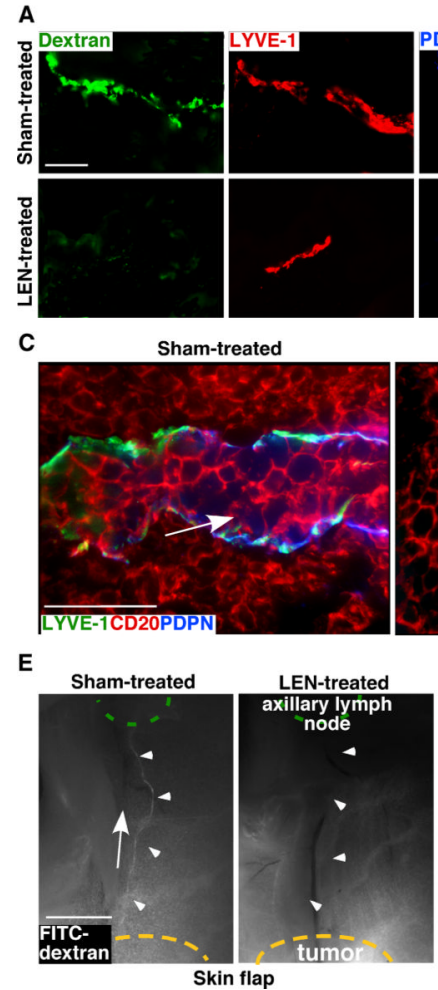

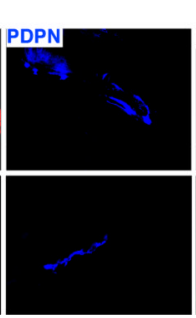

LEN-treated

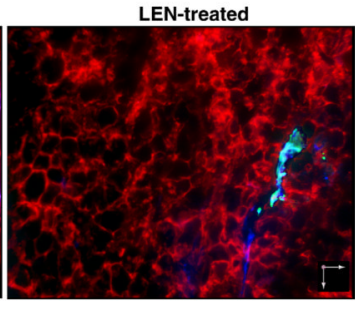

F

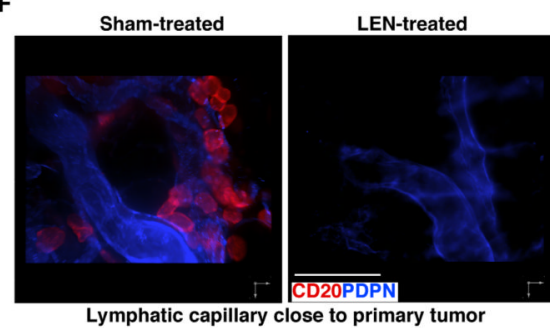

Figure 3.

LEN treatment results in non-functional tumor lymphatic vessels. A. MCL tumors immunostained for LYVE-1 and podoplanin (PDPN). The tumor tissues were collected 45 minutes after intratumor injection of FITC-dextran. Arrow indicates a FITC-dextran-filled lymphatic vessel. B. Percent of lymphatic vessels filled with FITC-dextran per $20 \times$ field in MCL tumors. Data represent the mean $\pm \operatorname{SEM}(n=6)$. C. Representative three-dimensional confocal images from sham- and LEN-treated tumor sections (1 $\mathrm{mm}$ in thickness) immunostained for lymphatic markers LYVE-1 and PDPN and MCL tumor marker CD20. Arrow indicates a tumor cell-containing lymphatic vessel. D. Percent of tumor cellcontaining lymphatic vessels in MCL tumors. Data represent the mean \pm SEM $(n=6)$. Scale bars, $50 \mu \mathrm{m}$. $* P<0.05$. E. Two hours after intratumor injection of FITC-dextran, fluorescence analysis of a whole-mount skin flap showed that the FITC-dextran was present in the collecting lymphatic vessel (arrowheads) of sham-treated, but not LEN-treated mice. Arrow marks the direction of lymph flow. Scale bar, $2 \mathrm{~mm}$. F. Reconstructed 3-dimenstional confocal images of PDPN ${ }^{+}$lymphatic vessels surrounding MCL xenografts. Scale bar, 50 $\mu \mathrm{m}$. 

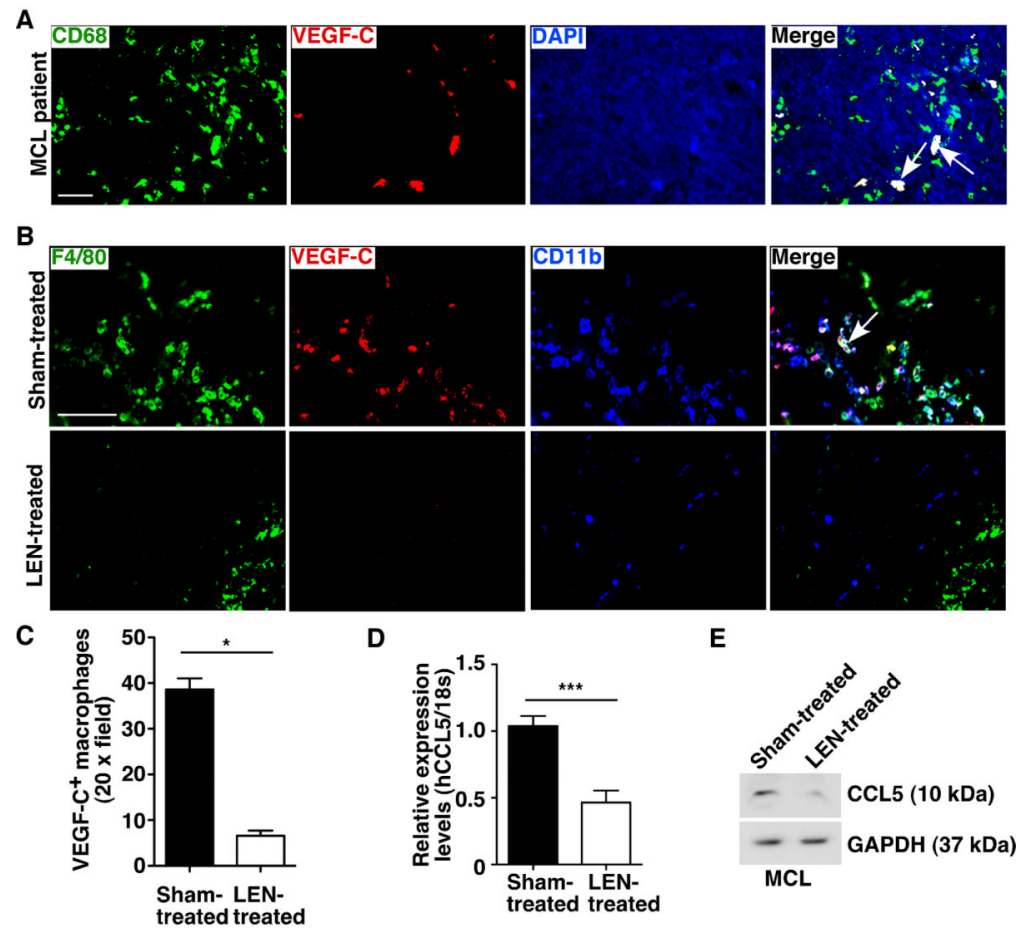

D

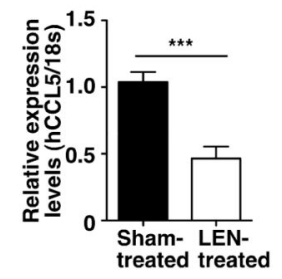

E

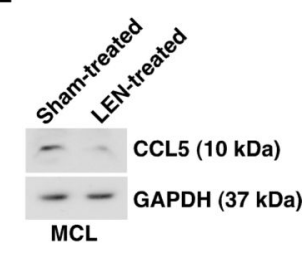

G Control
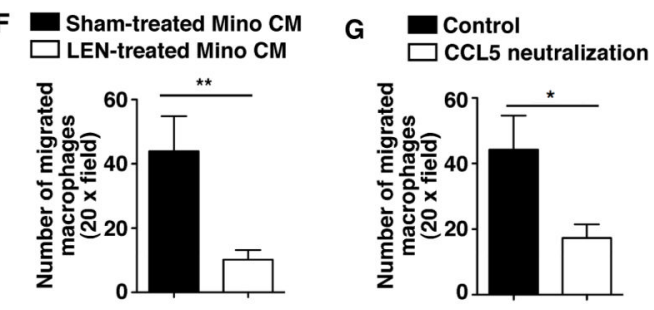

Figure 4.

MCL tumors contain VEGF-C-expressing macrophages and LEN reduces the number of tumor-associated macrophages. A. Immunofluorescent staining of CD68, a macrophage marker, and VEGF-C in human MCL tumor sections. Arrows indicate VEGF-C ${ }^{+}, \mathrm{CD}^{+}{ }^{+}$ macrophages. Scale bar, $100 \mu \mathrm{m}$. B. Immunofluorescent staining of VEGF-C and macrophage markers F4/80 and CD11b in the peripheral regions of sham- and LEN-treated mouse MCL tumors. Arrow indicates a VEGF-C ${ }^{+}$macrophage. Scale bar, $100 \mu \mathrm{m}$. C. $\mathrm{F} 4 / 80^{+}$, VEGF-C ${ }^{+}$, and $\mathrm{CD} 11 \mathrm{~b}^{+}$macrophages were quantified in randomly selected areas from the tumor peripheral regions. Data represent the mean $\pm \mathrm{SD}(\mathrm{n}=6, * P<0.05)$. D. Expression of human CCL5 in MCL tumor cells was analyzed by real-time PCR. Results represent relative units after normalized to the expression of 18S RNA. Data represent the mean $\pm \operatorname{SEM}(\mathrm{n}=3, * * * P<0.001)$ from three independent experiments. E. Immunoblot analysis of human CCL5 levels in MCL tumor cells. GAPDH is an internal control. Data are representative of three experiments. F. Macrophage migration measured in response to Mino cell conditioned media $(\mathrm{CM})$ with or without LEN treatment using a transwell assay. Results represent the mean $\pm \mathrm{SD}(\mathrm{n}=9)$ from three independent experiments. $* * P<0.01$. G.

Macrophage migration in response to sham-treated Mino cell conditioned media with a neutralizing antibody against CCL5 or isotype IgG (control). Results represent the mean \pm $\mathrm{SD}(\mathrm{n}=9)$ from three independent experiments. $* P<0.05$. 

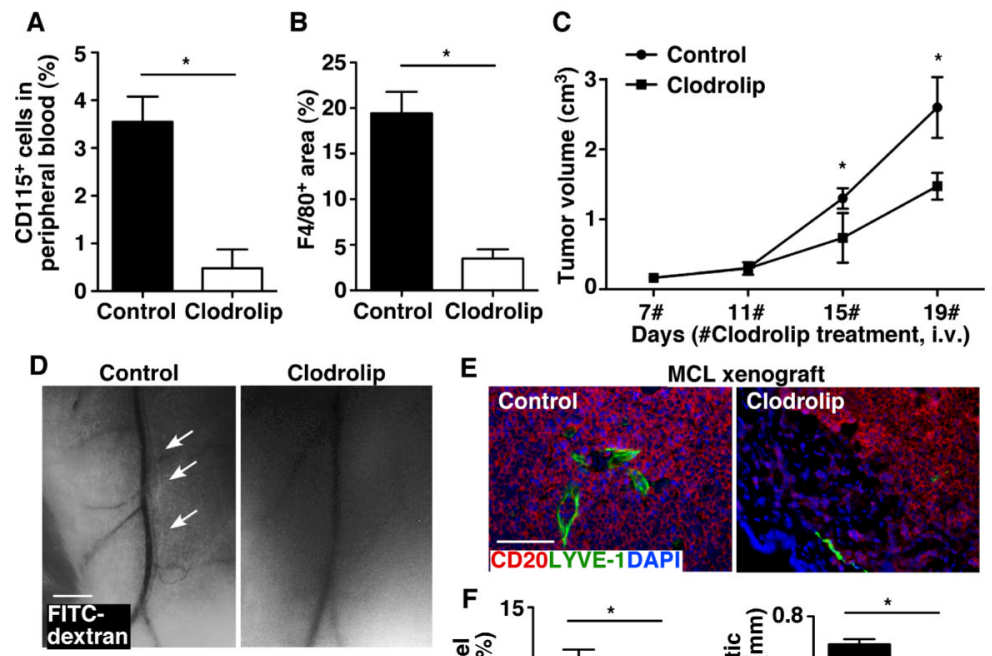

CL xenograft
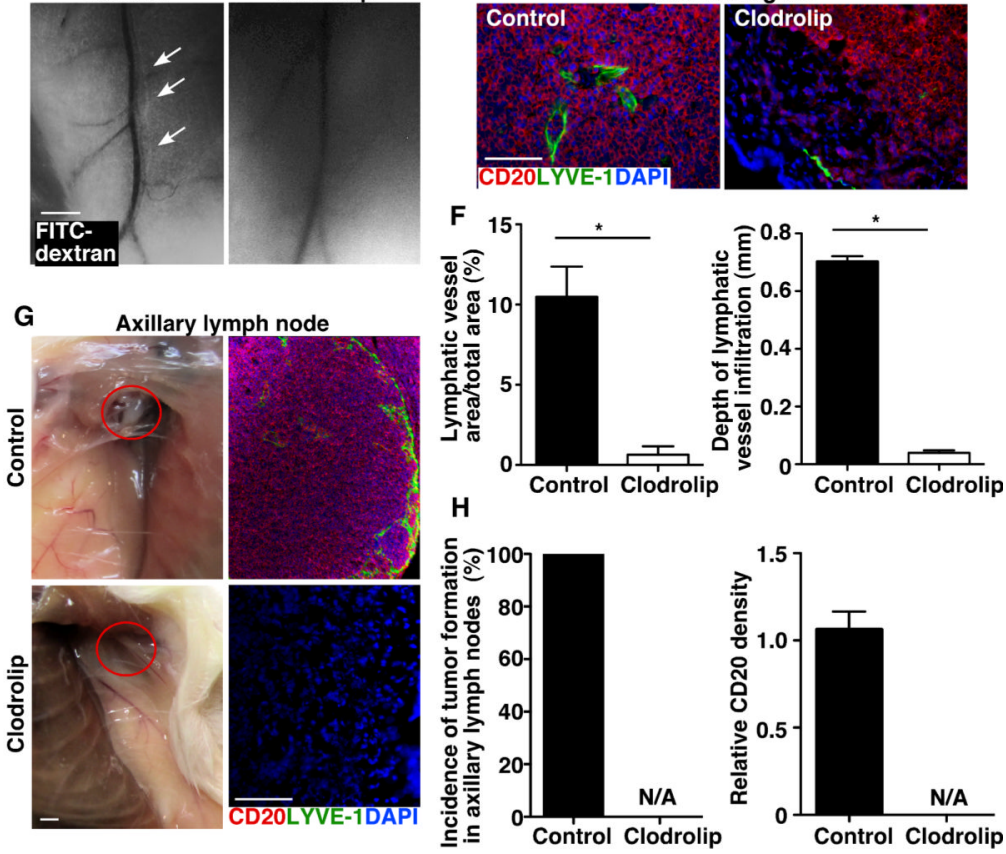

Figure 5.

Clodrolip reduces the number of tumor-associated macrophages, inhibits tumor lymphangiogenesis, and impairs MCL growth and dissemination. A. Flow cytometry of $\mathrm{CD} 115^{+}$monocytes/macrophages in the peripheral blood from control liposome- (control) or clodrolip-treated mice bearing MCL tumors. Data represent the mean $\pm \mathrm{SD}(\mathrm{n}=3)$. B. Quantification of F4/80 $0^{+}$cells from the peripheral regions of tumors. Data represent the mean $\pm \operatorname{SD}(n=6)$. C. Volume of MCL tumors. Data represent the mean $\pm S D(n=6)$. D. Lymphangiography of whole-mount skin flaps showed that the FITC-dextran was present in the collecting lymphatic vessel (arrows) of control-treated but not clodrolip-treated mice. Scale bar, $2 \mathrm{~mm}$. E-F. Representative immunostaining images and quantification of LYVE- $1^{+}$lymphatic vessels and infiltration depths in MCL xenografts treated with control liposomes or clodrolip. Scale bar, $100 \mu \mathrm{m}$. Lymphatic vessel density was determined from at least six representative images from each tumor. Data represent the mean $\pm S D(n=6)$. G. Gross analysis of axillary lymph nodes in control liposome- and clodrolip-treated mice bearing MCL tumors. Red circles highlight the axillary lymph node region. Scale bar, $5 \mathrm{~mm}$. Immunofluorescence staining for human CD20 and murine LYVE-1 of axillary lymph node sections. Scale bar, $100 \mu \mathrm{m}$. H. Quantification of disseminated CD20 ${ }^{+}$Mino cells in the axillary lymph nodes $(\mathrm{n}=6)$. Relative $\mathrm{CD} 20^{+}$positive staining density was presented as Mean $\pm \mathrm{SD}$ values $(\mathrm{n}=6) . * P<0.05$. 